\title{
On the Issue of Increasing Reliability of Electric Mining Machinery
}

\author{
Veniamin Kashirskikh ${ }^{1, *}$, Valery Zavyalov ${ }^{2,3}$, and Irina Semykina ${ }^{3}$ \\ ${ }^{1}$ T.F. Gorbachev Kuzbass State Technical University, Department of Electric Drive and Automation, \\ 650000, 28 Vesennyaya Street, Kemerovo, Russia \\ ${ }^{2}$ National Research Tomsk Polytechnic University, School of Energy \& Power Engineering, 634050, \\ 30 Lenin Avenue, Tomsk, Russia \\ ${ }^{3}$ Sevastopol State University, Institute of Nuclear Energy and Industry, 299053, 33 Universitetskaya \\ Street, Sevastopol, Russia
}

\begin{abstract}
The paper considers conditions of mining machinery electric drives operation and analyzes the causes of insufficient reliability. To improve the reliability and efficiency of induction motors the authors propose the computer system designed for dynamic identification of electric motors to monitor their parameters and variables, which are estimated on operating equipment in real time. Operation of the system allows calculating unmeasurable quantities and is based on the mathematical model of the induction motor as well as mathematical methods of estimation and the information contained in the measured phase voltages and currents of the stator. For dynamic mode the mathematical model of the motor's state and the measurement part were developed and certain results were obtained. Real-time information can be used for both control and management of electric motors, function testing, protection, forecasting as well as acceptance testing of electric motors in mining machinery electric drives to identify their individual data and quality control of the industrial processes in the manufacture or repair of motors.
\end{abstract}

\section{Introduction}

The efficiency of coal enterprises is heavily determined by the reliability and efficiency of electric mining machineries such as treatment and tunneling machines, scraper and belt conveyors, drilling rigs, excavators and other machines producing destruction and transportation of coal and rock. High reliability of underground mining machines which mainly use induction motor drives without speed controlling is a difficult task due to the severe operating conditions caused by the specific character of the process with randomly varying loads, frequent starts under load and overload of the actuator [1].

These factors result in transient processes in electric motors with high dynamics, significant mechanical vibration and shock loads in the transmission that lead to a deterioration of the stator winding insulation and intensive accumulation of fatigue damage

* Corresponding author: kash.veniamin@gmail.com 
in mechanical elements, premature wear, damage, accidents and, as a result, causing great economic damage. Electric motors are the main components of the drives which generate mechanical energy for mining machines movement and the destruction and displacement of the rock mass. Therefore special attention should be given to induction motor drives of mining machines in terms of increasing their functional reliability.

Currently, there is a promising trend in mining machinery to implement the controlled electric drives that deploy modern information systems for the state control and management, function testing and protection of the induction motor (IM) against emergency modes. The state of the IM is understood as some set of coordinates implying parameters, output, and intermediate variables and their derivatives, which vary in time as a function of input actions. It is known that the efficiency of control systems of electric drives depends on the current values of the electric motors electromagnetic parameters resistance, inductance and mutual inductance of the windings. The parameter values are listed in datasheets and since they are average calculated values, they can not be used for control in particular cases as accurate real values.

The electric motors' actual values depend on the operation mode and the thermal state. Thus, in the direct starting mode the rotor resistance can vary by more than 1.5 times, and inductance - by $30-40 \%$. The resistance of the stator winding can be changed during IM operation by $20-30 \%$, which is especially true for intermittent duty. Consequently, the current electric values should be estimated directly during operation. This becomes possible during dynamic motor identification. While the mining machine is working, it defines the current values of the parameters and the electromagnetic motor variables characterizing its state. The basis of dynamic identification, in this case, is the computer processing of the information contained in the voltages and currents of the motor based on a mathematical model of the motor and mathematical methods of identification.

The need for dynamic identification is based on the fact that most of the electromagnetic parameters and state variables of motors are not available through direct measurements. For example, for induction motors these are resistance and inductance of the rotor, magnetizing circuit inductance and flux linkage of stator and rotor, furthermore, in process of IM operation, the parameters of the stator also become inaccessible for direct measurement.

\section{Materials and Methods}

There is a significant number of publications on the identification of the individual parameters and state variables of electric motors for different modes as well as technical solutions for their implementation [2-11]. At the same time, there is a need to develop a comprehensive approach for dynamic identification methods of electric mining machinery to monitor their parameters and the state in order to use the information obtained in this case for control and management of electric motors, as well as for solving the tasks of function testing, protection, forecasting, and use on stage of acceptance testing of electric motors to identify their individual data and quality control of the industrial process in the manufacture or repair. For these purposes, it is necessary to develop methods having low sensitivity to the noise level as well as statistical characteristics and ensuring the stability of estimation calculation.

Dynamic identification of IM states and parameters requires the computer system that is based on the mathematical model of the motor, identification methods and measurement of available variables during operation and allows us to calculate the current values of the parameters and state variables in real-time that are not available for measurement. The theoretical basis for solving these problems is given in [12-16].

Taking into consideration all methods of dynamic identification described in the abovementioned references, the Recursive Least Squares Method (RLSM) and the 
Extended Kalman Filter (EKF) have been selected for identification of IM within the specified conditions.

\section{Results}

\subsection{Design of Estimator}

The equations of the two-phase generalized electric machine (GEM) [17-22] were used as a basis for the mathematical model of IM. This approach is widely applied for a variety of theoretical and practical problems and the model adequacy is proved by numerous practical implementations. Many publications noted [23-25] that the degree of accuracy for solutions obtained by GEM is sufficient for majority of practical purposes and depends mainly on the accuracy of the motor's parameters estimation.

The complex form of the IM model in the steady-state reference frame can be represented by a set of the following equations:

$$
\left\{\begin{array}{l}
\mathbf{u}_{1}=\mathbf{i}_{1} R_{1}+\boldsymbol{\Psi}_{1}^{\ell} \\
0=\mathbf{i}_{2} R_{2}+\boldsymbol{\Psi}_{2}^{\ell}-j p \omega_{r} \Psi_{2} \\
\boldsymbol{\Psi}_{1}=\mathbf{i}_{1} L_{1}+\mathbf{i}_{2} L_{m} \\
\boldsymbol{\Psi}_{2}=\mathbf{i}_{2} L_{2}+\mathbf{i}_{1} L_{m} \\
L_{1}=L_{1 \sigma}+L_{m} \\
L_{2}=L_{2 \sigma}+L_{m} \\
T=\frac{3}{2} p \operatorname{Im}\left(\Psi_{1}^{*} \mathbf{i}_{1}\right) \\
\boldsymbol{c}_{r}=\left(T+T_{L}\right) / J
\end{array}\right.
$$

where $\mathrm{u}_{1}, \mathrm{i}_{1}, \mathrm{i}_{2}, \Psi_{1}, \Psi_{2}$ - vectors of voltages, currents and flux linkages of stator and rotor windings respectively; $\omega_{r}$ - mechanical angular speed of rotor; $R_{1}, R_{2}, L_{1}, L_{2}$-resistance and inductance of the stator and rotor windings; $L_{m}$ - mutual inductance; $L_{1 \sigma}, L_{2 \sigma}$ - leakage inductance of stator and rotor windings; $T$ - electromagnetic torque; $T_{L}$ - load torque on shaft; $J$ - rotor inertia; $p$ - number of poles pair.

Hereinafter, subscripts 1 and 2 correspond to stator and rotor values respectively normalized to the stator. The superscript * indicates that the vector is conjugate, and the dot above a variable denotes its derivative. The assumptions of IM model correspond to the standard assumptions for GEM.

In static and dynamic modes of IM the measured values of voltages and currents contain different amounts of information about the motor's parameters and state variables, hence for these modes, different methods of identification were developed. As a result, based on IM model in which the state variables and measuring circuit variables was pointed out a set of methods based on RLSM and EFK was developed. They include algorithms for the estimation of the individual vector components of the parameter and the state variables of electric motors for static and dynamic modes, the algorithms for the preliminary identification of IM and software. The dynamic mode intensity is estimated by means of a numerical criterion that takes into account the power coefficient of the first harmonic of 
stator flux linkage $K_{p 1}$. Studies have shown that if $K_{p 1}>0.97$ the motor mode may be considered as static with the use of suitable algorithms.

Mathematical model of the IM with the state variables and measuring circuit variables used for identification by RLSM and EFK in static modes was obtained with the use of information about the parameters and IM state variables contained in the measured rotor speed, the phase voltages and currents of the stator. The IM model in static mode is the following equations:

$$
\begin{aligned}
& \Psi_{2 a}^{\&}=-\frac{R_{2}}{L_{2}} \Psi_{2 \alpha}-p \omega_{r} \Psi_{2 \beta}+\frac{R_{2} L_{m}}{L_{2}} i_{1 \alpha} ; \\
& \Psi_{2 \beta}^{\&}=-\frac{R_{2}}{L_{2}} \Psi_{2 \beta}+p \omega_{r} \Psi_{2 \alpha}+\frac{R_{2} L_{m}}{L_{2}} i_{1 \beta},
\end{aligned}
$$

where subscripts $\alpha$ and $\beta$ correspond to vectors' components in the steady-state reference frame, and the identifiable vector of the motor's parameters and the state variables in static mode is the following:

$$
\theta_{s}=\left[\begin{array}{lllllll}
\Psi_{1} & \Psi_{m} & \Psi_{2} & R_{1} & L_{1 \sigma} & L_{2} & L_{m}
\end{array}\right]^{T}
$$

where $\Psi_{1}, \Psi_{2}, \Psi_{m}$ - vector magnitude of stator, rotor and mutual flux linkage respectively.

Unlike static mode, when IM operates in dynamic mode it is possible to identify simultaneously the rotor resistance and mechanical angular speed of rotor. Identification of IM parameters and state variables in this mode is based on the use of EFK and the information contained only in the phase voltages and currents of the stator. This procedure involves the expansion of the motor's state vector by introducing the load torque and resistance of stator and rotor:

$$
x_{e}=\left[\begin{array}{llllll}
\Psi_{2 \alpha} & \Psi_{2 \beta} & \omega_{r} & R_{1} & R_{2} & T_{L}
\end{array}\right]^{T},
$$

and the conversion of original equations (1) in difference equations thus IM model becomes the following equations:

$$
f\left(x_{e}, z\right)=\left[\begin{array}{c}
f_{1} \\
f_{2} \\
f_{3} \\
f_{4} \\
f_{5} \\
f_{6}
\end{array}\right]=\left[\begin{array}{c}
\Psi_{2 \alpha}+\left(-\frac{R_{2}}{L_{2}} \Psi_{2 \alpha}-p \omega_{r} \Psi_{2 \beta}+\frac{R_{2} L_{m}}{L_{2}} i_{1 \alpha}\right) T_{s} \\
\Psi_{2 \beta}+\left(-\frac{R_{2}}{L_{2}} \Psi_{2 \beta}+p \omega_{r} \Psi_{2 \alpha}+\frac{R_{2} L_{m}}{L_{2}} i_{1 \beta}\right) T_{s} \\
\omega_{r}+\frac{1}{J}\left(\frac{3}{2} \frac{L_{m}}{L_{2}} p\left(\Psi_{2 \alpha} i_{1 \beta}-\Psi_{2 \beta} i_{1 \alpha}\right)-T_{L}\right) T_{s} \\
R_{1} \\
R_{2} \\
T_{L}
\end{array}\right] .
$$

where $T_{s}$ - sampling time. 
A mathematical model of the measuring circuit was obtained by simultaneous solution of the equations of rotor flux linkage from (1) and the equations (2) for the IM state model for the EFK-based rotor resistance estimation:

$$
\begin{aligned}
& y=\left[\begin{array}{ll}
u_{1 \alpha} & u_{1 \beta}
\end{array}\right]^{T} ; \\
& h\left(x_{e}, u\right)=\left[\begin{array}{l}
h_{1} \\
h_{2}
\end{array}\right]=\left[\begin{array}{l}
-\frac{R_{2} L_{m}}{L_{2}^{2}} \Psi_{2 \alpha}-p \omega_{r} \frac{L_{m}}{L_{2}} \Psi_{2 \beta}+\left(R_{1}+R_{2} \frac{L_{m}^{2}}{L_{2}^{2}}\right) i_{1 \alpha}+\frac{L_{1} L_{2}-L_{m}^{2}}{L_{2}} \mathbb{1}_{1 \alpha}^{\&} \\
-\frac{R_{2} L_{m}}{L_{2}^{2}} \Psi_{2 \beta}+p \omega_{r} \frac{L_{m}}{L_{2}} \Psi_{2 \alpha}+\left(R_{1}+R_{2} \frac{L_{m}^{2}}{L_{2}^{2}}\right) i_{1 \beta}+\frac{L_{1} L_{2}-L_{m}^{2}}{L_{2}} i_{1 \beta}^{\&}
\end{array}\right] .
\end{aligned}
$$

On the basis of EFK the identifiable vector of the motor's parameters and the state variables in dynamic mode is:

$$
\theta_{d}=\left[\begin{array}{llllll}
\Psi_{2 \alpha} & \Psi_{2 \beta} & \omega_{r} & R_{1} & R_{2} & T_{L}
\end{array}\right]^{T} .
$$

Other IM parameters are estimated at the stage of preliminary identification based on the data obtained at IM starting without load and also at IM operating in the static mode with idle speed.

To estimate the resistance of the stator winding we developed a method based on the introduction of the permanent components in phase voltages followed by the processing of the data from the measuring system used in the IM dynamic identification [26]. If the value of resistance of the stator winding is known it is relatively easy to calculate the stator flux linkage with damping instead of integration, which can give a large error due to noise in the measuring system [27].

The shape of the IM magnetization curve in the idle mode is estimated by comparing the time of current and stator flux linkages [28]. To exclude the errors caused by losses in IM, the maximum value of current and stator flux are aligned in time. Current and flux are tabulated in time during one period to obtain an array of data that can be presented as a table or graph of the IM magnetization curve. Estimation of the rotor moment of inertia is also carried out during the pre-identification by a known method.

To perform the estimation process the software of algorithms contains coordinate converter of stator voltages and currents from the three-phase system of coordinates in the two-phase system, the calculator for the derivative components of stator currents, calculators for IM parameters and variables, which are based on the use of RLSM, EFK algorithms and other calculated dependencies.

\subsection{Estimation Results}

The procedure of dynamic identification was carried out for a number of IM. For example, Figure 1 - Figure 4 show the process of parameters estimation in the static mode for IM of 4A90L4Y3 series. Figure 5 shows the estimation process of the mechanical angular rotor speed in the dynamic mode for the same motor and Figure 6 shows measured data for comparison.

The estimation accuracy of IM parameters and variables was proved by comparative analysis of obtained results with measured data and information from motors' datasheets. 


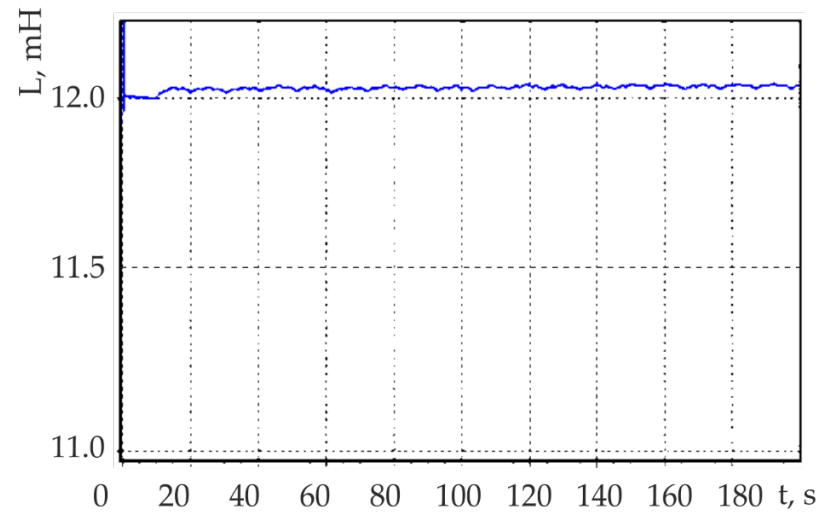

Fig. 1. The estimation process of leakage inductance of the stator winding.

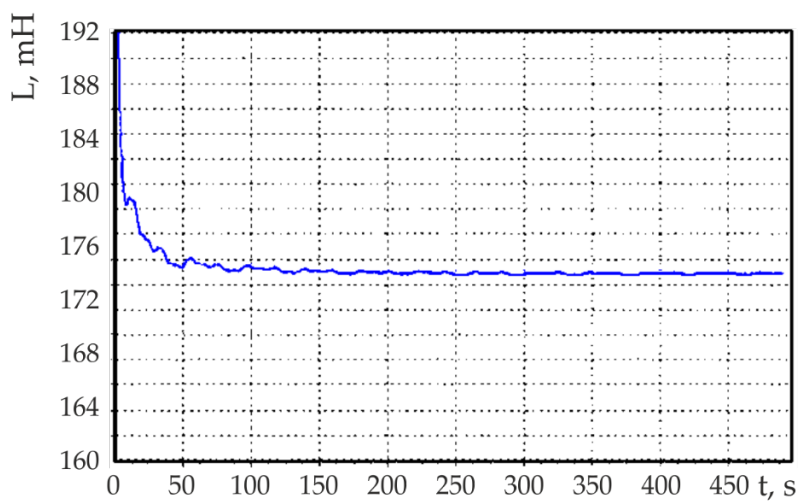

Fig. 2. The estimation process of mutual inductance.

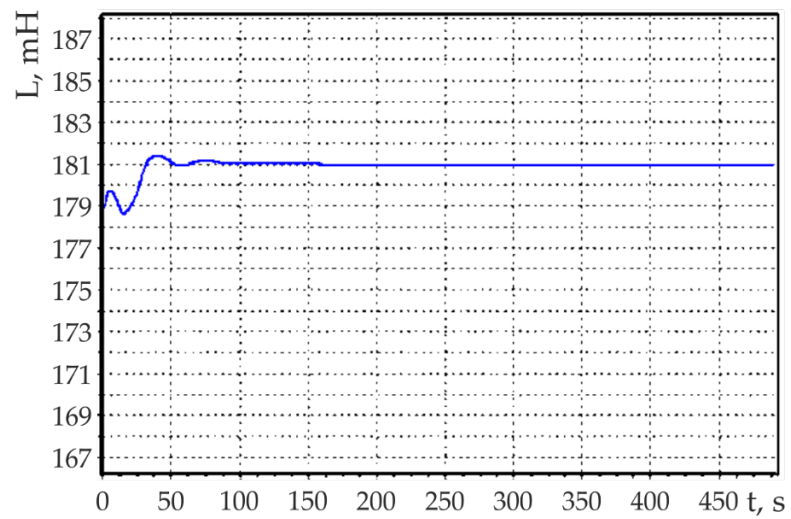

Fig. 3. The estimation process of the rotor inductance. 


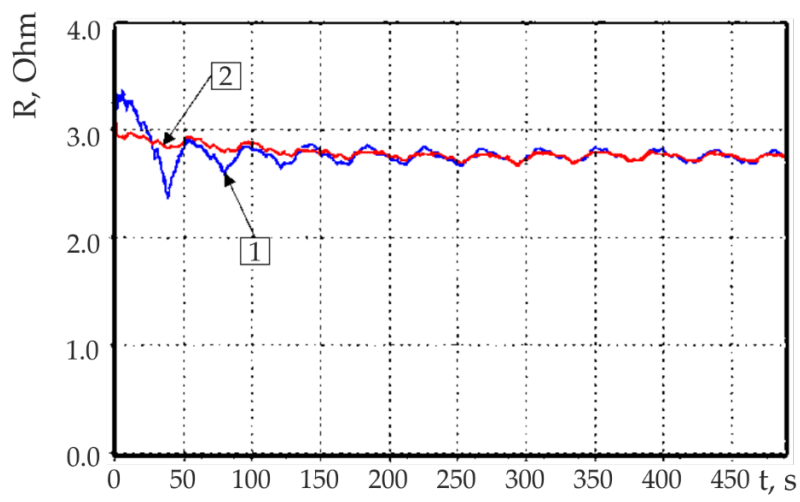

Fig. 4. The estimation process of the rotor resistance on the basis of EFK: (1) the analytical solution of the original differential equation (2) the approximation of the equation based on numerical Euler method.

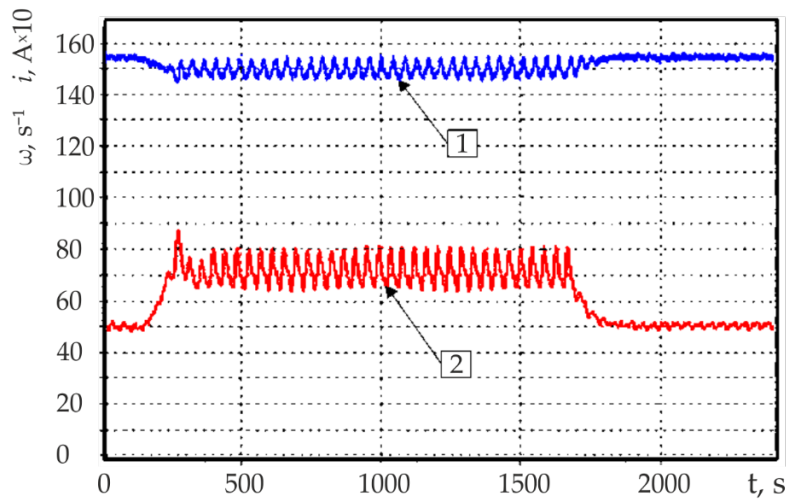

Fig. 5. The estimation process of the mechanical angular rotor speed (1) and the measured stator current (2).

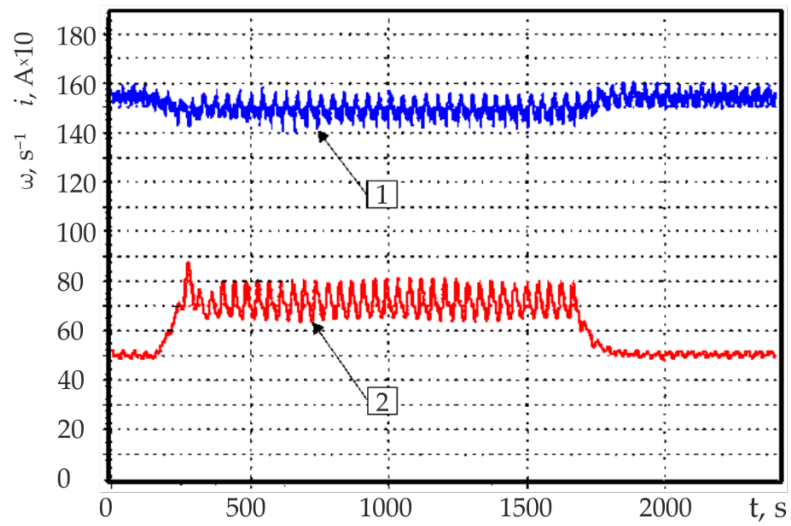

Fig. 6. The measured mechanical angular rotor speed (1) and the measured stator current (2). 
The comparative data of IM parameters estimated in the static mode for IM of 4A90L4Y3, 4AM80A4CY1, 4AMX90L2Y3, and AIM-M80B2Y2.5 series is shown in Table 1, where symbol $\mathrm{R}_{2}$ ' corresponds to the estimation or rotor resistance obtained on the basis of EFK and $\mathrm{R}_{2}$ is the RLSM based estimation.

Table 1. Comparative data for induction motors.

\begin{tabular}{|c|c|c|c|c|c|c|c|}
\hline \multirow{2}{*}{ Motor Type } & \multirow{2}{*}{ Data Source } & \multicolumn{6}{|c|}{ Parameters } \\
\hline & & $L_{1 \sigma}$ & $L_{1}$ & $L_{m}$ & $L_{2}$ & $R_{2}$ & $R_{2}^{\prime}$ \\
\hline \multirow{3}{*}{ 4A90L4Y3 } & Datasheet & 0.010 & 0.303 & 0.263 & 0.311 & \multicolumn{2}{|l|}{2.63} \\
\hline & Estimation & 0.012 & 0.189 & 0.175 & 0.181 & 2.71 & 2.75 \\
\hline & Measurement & - & 0.202 & - & - & \multicolumn{2}{|l|}{-} \\
\hline \multirow{3}{*}{ 4AM80A4CY1 } & Datasheet & 0.01 & 0.444 & 0.434 & 0.465 & \multicolumn{2}{|l|}{5.5} \\
\hline & Estimation & 0.01 & 0.285 & 0.275 & 0.282 & 5.0 & 5.0 \\
\hline & Measurement & - & 0.270 & - & - & \multicolumn{2}{|l|}{-} \\
\hline \multirow{3}{*}{ 4AMX90L2Y3 } & Datasheet & 0.003 & 0.393 & 0.39 & 0.401 & \multicolumn{2}{|l|}{1.69} \\
\hline & Estimation & 0.003 & 0.198 & 0.195 & 0.2 & 1.00 & 1.02 \\
\hline & Measurement & - & 0.203 & - & - & \multicolumn{2}{|l|}{-} \\
\hline \multirow{3}{*}{ AIM-M80B2Y2.5 } & Datasheet & - & - & - & - & \multicolumn{2}{|l|}{-} \\
\hline & Estimation & 0.008 & 0.368 & 0.36 & 0.37 & 1.32 & 1.30 \\
\hline & Measurement & - & 0.37 & - & - & \multicolumn{2}{|l|}{-} \\
\hline
\end{tabular}

\subsection{Computer System}

Based on the described methods of dynamic identification, the computer system for electric drives fault diagnostic was developed that aimed at using as a supplementary tool for condition monitored maintenance of mining machinery as part of planned preventive maintenance. The structure of the computer system is shown in Figure 7. This structure is generalized and can be implemented to electric drives for different types of mining machines. If these electric drives are linked via machinery mechanics, it has to expand similarly by adding as many parts as the number of drives.

The system structure includes the induction motor drive consisting of the induction motor supplied by electricity with a power converter and working under a control system, which might vary in a wield range depending on the type of electric mining machinery. Other elements of the system were developed with a diagnostic meaning. They are the observer with RLSM and EFK algorithms, the predictor and three analyzers of technical state for the control system, the power converter, and the electric drive at whole. The key role in the computer system operation is played by command variable vector $\mathbf{u}$, the motor state vector $\mathbf{x}$ and estimated IM parameters and variables.

The observer provides the real-time estimation of the electromagnetic and mechanical variables of the electric drive using the information contained in the measured currents, voltages of the motor as well as velocities and angular shaft position of electric drive mechanics. For one-step ahead predicting of the motor state vector $\hat{\mathbf{x}}$ according to the history of its changing, the predictor is used. This component of the computer system builds on the basis of an artificial neural network. 


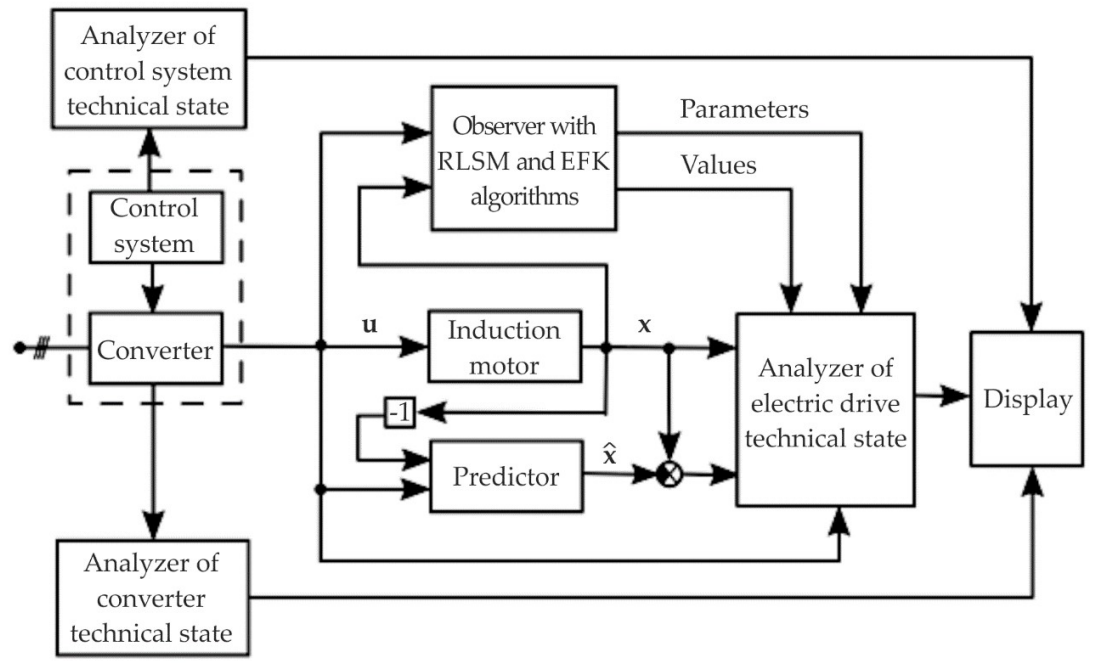

Fig. 7. Structure of the computer system for electric drives fault diagnostic.

The estimated IM parameters and variables, the command variable vector $\mathbf{u}$ and the difference between vectors $\mathbf{x}$ and $\hat{\mathbf{x}}$ use as impute signals for the analyzer of electric drive technical state. This analyzer provides computer analysis of the incoming information and performs the recognition of the technical condition of the operating electric drive by a number of signs with displaying the resulting information.

\section{Discussion and Conclusions}

Analysis of the research results led to the following conclusions. The developed identification algorithms have the relatively equal duration of the estimation process that is close to one period of the supply voltage lasting $20 \mathrm{~ms}$ for the stator leakage inductance, the rotor and the mutual flux linkages. The duration of the estimation process for the rotor inductance and the mutual inductance is in the range of 40-50 ms. The value of the rotor resistance on the basis of the second EFK decision option is also assessed within $20 \mathrm{~ms}$, and this option is adopted as the basic one. When using the first option, as well as in assessing the value of the rotor resistance using RLSM, the duration of the estimation process is $120 \mathrm{~ms}$. The studies found that the statistical properties of the noise in measuring system used for the dynamic identification of electric motors fully meet the requirements of the methods applied [29]. At the same time, computational processes have the required stability level.

The data obtained for IM identification is much closer to the measured values than the data in the datasheets, which is calculated for the series. Because of the high information content of the stator currents and voltages the estimates are more accurate in the dynamic mode. Studies have shown that in this case, the relative error does not exceed $3 \%$. These methods allow estimating the current values of the IM parameters and state variables in real-time basing on the information from sensors which measure the phase currents and the stator voltage. For IM working in static mode, it is necessary to measure the angular speed of the rotor.

Thus, the methods developed for dynamic identification can be recommended for IM state control, as well as for the use in functional testing of induction motors and acceptance 
testing of IM after their manufacture and repair. The designed computer system for electric drives fault diagnostic could be used as a supplementary tool for condition monitored maintenance of mining machinery as part of planned preventive maintenance.

This approach is one of the ways of enhancing the efficiency and reliability of induction motor drive for electric mining machinery.

\section{References}

1. I. V. Breido, I. Yu. Semykina, G. S. Nurmaganbetova, Bulletin of the Tomsk Polytechnic University, Geo Assets Engineering, 329(2), 65-73 (2018)

2. F. Alonge, F. D'Ippolo, La.S. Barbera, F.M. Raimondi, Procedings of the 1998 IEEE Interniteanal Conference on Control Applications Trieste, 1, 491-496 (1998)

3. S. Jangjit, P. Laohachai, Journal of Electrical Engineering \& Technology, 4(3), 360-364 (2009)

4. K. Kampisios, P. Zanchetta, C. Gerada, A. Trentin, Proceedings of the Industrial Applications Society Annual Meeting, 1, 78 (2008)

5. S. Aksoy, A. Muhurcu, H. Kizmaz, Proceedings of Modern Electric Power Systems,

1, 5 (2010)

6. P. Simonik, P. Hudecek, P. Palacky, D. Slivka, Progress in Electromagnetics Research Symposium Proceedings, 1, 165 (2012)

7. R. Giri, A. Chowdhury, A. Ghosh, B. K. Panigrahi, Proceedings of SEMCCO, 1, 523530 (2010)

8. N. A. Korolev, S. V. Solovev, IOP Conference Series: Materials Science and Engineering, 177(1), (2017)

9. H. A. Toliyat, E. Levi, Munpreet Kaur Raina, IEEE Power Engineering Review, 22, 52$52(2002)$

10. Li Shanshan, Journal of Physics: Conference Series, 1302, 022054 (2019)

11. D. K. Chaturvedi, M. P. Singh, Journal of the Institution of Engineers (India): Series B, 100, 343-347 (2019)

12. P. Eikhoff, Fundamentals of control systems identification. Estimation of parameters and states (Mir, Moscow, 1975)

13. J. Medich, Statistical Optimal Linear Estimators and Control (Energiya, Moscow, Russia, 1973)

17. C. B. Speedy, R. F. Brown, G. C. Goodwin, Control Theory: Identification and optimal control (Mir, Moscow, 1973)

15. A. A. Krassovskiy, Guidebook on the automated control theory (Nauka, Moscow, Russia, 1987)

16. R. Lee, Optimal estimators, determining characteristics and control (Nauka, Moscow, Russia, 1996)

17. G. Kron, Tensor analysis of networks (J. Wiley \& Sons Chapman \& Hall: London, UK, 1939).

18. P. K. Kovacs, E. Racz, Transient Phenomena in Electrical Machines (Elsevier Science Publishers, Amsterdam, the Netherlands, 1984)

19. D. C. S. White, H. H. Woodson, Electromechanical Energy Conversion (John Wiley \& Sons, New York, USA, 1959)

20. N. Hancock, Matrix analysis of electrical machinery (Energiya, Moscow, 1967) 
21. R. V. Filtz, Mathematical bases of electromechanical converters theory (Naukova Dumka, Kiyev, 1979)

22. V. I. Klyuchev, Electric Drive Theory (Energoatomizdat, Moscow, 2001)

23. I. P. Kopylov, Mathematical modelling of electric machines (Vysshaya Shkola, Moscow, 2001)

24. G. S. Sipaylov, A.V. Loos, Mathematical modelling of electric machines (Vysshaya Shkola, Moscow, 1980)

25. B. Ya. Stariko, I. L. Azarkh, Z. M. Rabinovich, Induction motor for shearers (Nedra, Moscow, 1981)

26. V. G. Kashirskikh, A. V. Nesterovskiy, A. N. Gargayev, A method for determining the stator resistance of the induction motor, Russian pat. No. 2406096, 34 (2010)

27. V. G. Kashirskikh, A. V. Nesterovskiy, A. N. Gargayev, A method for determining the induction motor stator flux linkage, Russian pat. No 2439599, 1 (2012)

28. V. G. Kashirskikh, V. M. Zavyalov, D. V. Sokolov, Bulletin of the Kuzbass State Technical University, 2, 14-16 (2002)

29. V. G. Kashirskikh, V. M. Zavyalov, A. V. Nesterovskiy, Bulletin of the Kuzbass State Technical University, 2, 12-14 (2003) 